\title{
IMAGE, DIAGRAMME ET MÉTAPHORE
}

\author{
A propos de l'icône chez C.S. Peirce
}

\author{
Philippe Verhaegen'
}

\section{Problèmes métaphoriques}

\section{Un procédé bien ordinaire}

Il est courant de rencontrer dans nos journaux des expressions nous annonçant que "La guerre des honoraires médicaux" est imminente, que le franc (français) "est dos au mark", que "Des maires à l'État, chacun se renvoie la balle" et que les écologistes "vont boucler leur tour de France". Aucun sujet d'actualité ne semble pouvoir échapper à cette emprise métaphorique: même la météo nous annonce que mardi, on doit s'attendre à une "nouvelle vague de froid".

Mais la métaphore est tout autant à l'œuvre dans les différentes formes de discours scientifique. Ainsi les sciences de la communication n'hésitent pas à parler de nos interactions en termes de "modèle de l'orchestre" ou de "modèle télégraphique". Des expressions de ce type sont présentes dans toute la littérature scientifique, sans exception: le physicien nous entretient de la théorie du "big bang", le neurologue de "la machine cérébrale", le biologiste du "codage génétique" ou de la "double hélice de l'ADN", etc. Rien d'étonnant alors à ce que

1 Chargé d'enseignement au Département de communication de l'Université catholique de Louvain. Responsable de l'Unité de recherche en communication (RECO).

Recherches en communication, $\mathrm{n}^{\circ} 1$ (1994). 
la vulgarisation scientifique s'en empare elle aussi, comme en témoigne l'extrait suivant: "du Big Bang jusqu'aux particules les plus fugaces, les tentacules de la pieuvre «Physique» se déploient aux deux extrémités du temp"".

Qu'est-ce qui justifie la fréquence d'emploi d'un tel procédé qui, somme toute, ne constitue qu'une des figures parmi toutes celles que la rhétorique met à notre disposition? Remplirait-elle un rôle particulièrement important dans nos systèmes sémiotiques?

\section{Un processus complexe}

Compte tenu de l'ampleur de la littérature traitant de la métaphore, examinons brièvement quelques "paradigmes" qui ont soustendu son analyse.

Il y a tout d'abord la métaphore "fondamentale", celle qui est postulée comme étant à la base de tout processus sémiotique. Cette thèse pourrait être soutenue par quelqu'un comme Jakobson. C'est à lui, en effet, que revient le mérite d'avoir proposé de rapprocher la notion de métaphore de celle de système chez Saussure. Tirant profit d'études portant sur les troubles du langage, il introduit la notion de métaphore -ou plus exactement de procès métaphorique-comme désignant, à côté de la métonymie, l'un des deux processus à l'œuvre dans l'expression linguistique.

Parler, dit-il, implique pour les locuteurs une activité de sélection d'entités linguistiques et leur combinaison en unités linguistiques d'un plus haut degré de complexité ${ }^{1}$. La première opération porte sur des entités associées dans le code par différents degrés de similarité et formant des séries mnémoniques virtuelles (des groupes de substitution) dans lesquelles le locuteur peut puiser. La seconde opération unit deux ou plusieurs termes également présents dans une série effective, chacun d'eux formant en quelque sorte le contexte de l'autre. Les constituants d'un contexte ont un statut de contiguïté.

Sur cette base, Jakobson constate que l'aphasique est quelqu'un qui a perdu la capacité d'associer des termes tantôt par similarité, tantôt par contiguïté. Dans les "troubles de la similarité", le malade a perdu la capacité d'effectuer des opérations métalinguistiques lui permettant, par exemple, de considérer la circonlocution personne non mariée comme équivalente au mot célibataire. Il ne peut donc plus

1 R. JaKoBSON, Deux aspects du langage et deux types d'aphasie, Essais de linguistique générale, Paris, Éd. de Minuit, 1963, p. 45-46. 
développer un discours où un thème en amène un autre par similarité. Bref, dans ce type d'aphasie, le procès métaphorique est amoindri ou totalement bloqué. A l'opposé, dans le trouble de la contiguïté, Jakobson parlera d'altération plus ou moins grave de la faculté de combinaison et de contexture, c'est-à-dire le pouvoir pour le malade de maintenir la hiérarchie des unités linguistiques. Cette fois, c'est le procès métonymique qui est mis en défaut.

Métaphore et métonymie constituent, pour Jakobson, deux processus fondamentaux de l'expression (verbale, iconique, psychique,...), processus nécessaires à la sélection et à la combinaison des unités sémiotiques. Deleuze et Guattari soutiennent exactement la thèse inverse.

Dans Mille Plateaux, ceux-ci avancent tout d'abord que le langage ne serait pas transmission d'information mais bien émission et réception de "mots d'ordre": "plutôt que le sens commun, faculté qui centraliserait les informations, il faut définir une abominable faculté qui consiste à émettre, recevoir et transmettre les mots d'ordre". Ceuxci seraient coextensifs au langage:

"si le langage semble toujours supposer le langage, si l'on ne peut pas fixer un point de départ non linguistique, c'est parce que le langage ne s'établit pas entre quelque chose de vu (ou de senti) et quelque chose de dit, mais va toujours d'un dire à un dire. (...) Le «premier» langage, ou plutôt la première détermination qui remplit le langage, ce n'est pas le trope ou la métaphore, c'est le discours indirect. L'importance qu'on a voulu donner à la métaphore, à la métonymie se révèle ruineuse pour l'étude du langage. Métaphores et métonymies sont seulement des effets, qui n'appartiennent au langage que dans le cas où ils supposent déjà le discours indirect" 1 .

Contrairement à Jakobson, la position de Deleuze et Guattari privilégie la dimension pragmatique du langage. En dehors de celleci, il n'y aurait pas de langage à proprement parler mais, par contre, la production de tropes demeurerait possible:

"Benveniste nie que l'abeille ait un langage, bien qu'elle dispose d'un encodage organique, et se serve même de tropes. Elle n'a pas de langage, parce qu'elle est capable de communiquer ce qu'elle a vu, mais non pas de transmettre ce qu'on lui a

1 G. Deleuze et F. GuatTARI, Postulats de la linguistique, Mille plateaux, Paris, Éd. de Minuit, 1980, p. 95-98. 
communiqué. (...) Le langage ne se contente pas d'aller d'un premier à un second, de quelqu'un qui a vu à quelqu'un qui n'a pas vu, mais va nécessairement d'un second à un troisième, ni l'un ni l'autre n'ayant vu. C'est en ce sens que le langage est transmission du mot fonctionnant comme mot d'ordre, et non pas communication d'un signe comme information. Le langage est une carte et non pas un calque"'.

La métaphore n'est pas une forme «première» du langage parce qu'elle ne présuppose pas le discours indirect. Toutefois, le propos de Deleuze et Guattari n'est pas prioritairement de parler de métaphore mais de mettre en cause une conception de la communication comme transmission d'information. Si l'on dit du dormeur que ses rêves sont de nature métaphorique, pour ce dernier, il n'en est rien. Enfermé dans son univers onirique, il croit parler le monde. Ce ne sera qu' une fois en situation de veille que l'écart entre monde imaginé et monde réel lui apparaîtra. Par conséquent, parler de métaphore c'est se placer dans un métalangage où celle-ci ne joue plus. Mais inversement cela signifie que tout langage peut être dit métaphorique: il suffit de trouver un métalangage capable de l'exprimer. C'est pourquoi, me semble-t-il, Deleuze et Guattari considèrent à la fois la danse des abeilles comme un non-langage -il ne va pas d'un dire à un dire- et comme se servant de tropes (du moins tel qu'on peut l'exprimer dans le métalangage humain).

Autrement dit, la métaphore doit, sur un plan pragmatique, se faire oublier. Ou bien, elle est au fondement de l'échange mais reste inaccessible tant que cet échange n'est pas passé par un méta-niveau, ou bien elle est mise en épingle mais alors elle ne joue plus que comme concept rhétorique. Quoiqu'il en soit, il semble bien qu'il faille s'accommoder d'un tel paradoxe. Le rapprochement des thèses respectives de Jakobson et de Deleuze et Guattari en donne un premier aperçu. Pour ma part, cela signifie qu'il faut tenter de définir la métaphore d'un point de vue tant sémiotique que pragmatique. A ma connaissance, ce travail reste à accomplir.

Ces deux thèses nous montrent également combien la métaphore est une notion nomade. Du domaine restreint de l'art oratoire et de la Poétique d'Aristote aux troubles de l'aphasie, la métaphore voyage et se transforme jusqu'à s'infiltrer dans notre quotidien. Une chose est sûre: la métaphore ne peut plus être considérée comme un simple ar-

1 Ibidem. 
tifice de l'expression verbale confiné à l'exercice de stratégies oratoires.

Au-delà des dimensions sémiotiques et pragmatiques évoquées ci-dessus, la métaphore est régulièrement citée comme participant activement à nos processus cognitifs. Cet aspect, quoique déjà présent chez Jakobson, a été maintes fois soulevé depuis. Ainsi, Lakoff et Johnson soutiennent que notre système conceptuel ordinaire -celui qui nous sert à penser et à agir- serait de nature fondamentalement métaphorique. Le concept de "discussion", par exemple, serait compris grâce à ce que les auteurs appellent une métaphore conceptuelle du type "la discussion, c'est la guerre", métaphore qui se reflète dans notre langage quotidien. "L'essence d'une métaphore, avancent les auteurs, est qu'elle permet de comprendre quelque chose (et d'en faire l'expérience) en termes de quelque chose d'autre"1.

Procédé rhétorique, processus cognitif ou mode d'interaction, la métaphore focalise des interrogations qui animent actuellement les sciences de la communication. Pour en rendre compte, on ne dispose pas d'un cadre théorique bien établi. Dès lors, je me propose dans les lignes qui vont suivre d'esquisser quelques propositions en ce sens. Je baserai mon propos sur certaines suggestions issues des conceptions sémiotiques et logiques de C.S. Peirce ${ }^{2}$. Les écrits de celui-ci posant encore nombre de problèmes d'interprétation, il me faudra préciser d'abord la façon dont je les comprends.

\section{La métaphore dans le système sémio-pragmatique de Peirce}

Chez Peirce tout processus sémiotique doit être considéré en rapport avec le fonctionnement global du signe. La métaphore ne constitue donc pas un type ou une classe de signes particuliers mais plutôt une opération, un mode de production du sens. Peirce la considère comme un des trois processus iconiques au côté de l'image et du dia-

1 G. LAKOFF ET M. JoHnSon, Les métaphores dans la vie quotidienne, Paris, Éd. de Minuit, 1985, p. 15.

2 C.S. PEIRCE, Collected papers of Charles Sanders Peirce, Harvard University press, Cambridge, 8 volumes, 1932-1958. Suivant l'usage qui s'est imposé en la matière, il est fait référence aux $\mathrm{CP}$ en usant d'une double numérotation séparée par un point. Le premier nombre indique le volume et le second, le paragraphe auquel il est fait allusion. Cette référence est suivie par celle de la traduction française quand elle existe (cf. C.S. PEIRCE, Écrits sur le signe, Paris, Seuil, 1978). 
gramme. Avant d'approfondir cet aspect, précisons les lignes de force de la sémiotique peircienne.

\section{Signe et sens}

Pour Peirce, un signe (ou representamen) c'est "quelque chose qui tient lieu pour quelqu'un de quelque chose sous quelque rapport ou à quelque titre. Il s'adresse à quelqu'un, c'est-à-dire crée dans l'esprit de cette personne un signe équivalent ou peut-être plus développé. Ce signe qu'il crée, je l'appelle l'interprétant du premier signe. Ce signe tient lieu de quelque chose: de son objet"1. Representamen, objet et interprétant constituent les trois dimensions du signe qui sont unies par une relation triadique dite "authentique" parce qu'elle n'est pas réductible à des relations entre pairs.

Le sens d'un signe n'est pas décodable dans la mesure où il ne peut être défini par un simple recours à un lexique et à des règles d'agencements préexistants. Le sens chez Peirce est pragmatique: "un signe est d'abord ce qu'il fait, et ce qu'il fait est sa signification, autrement dit la règle de l'action" 2 . Le signe serait-il dès lors "pragmatique"? Pas exactement -et c'est là sans doute l'originalité du système peircien: un signe est à la fois sémiotique et pragmatique. Voyons cela.

Pour comprendre un signe, l'interprète dispose d'interprétants, c'est-à-dire de signes intermédiaires lui permettant de rapporter le representamen perçu à l'objet qu'il est censé représenter. A priori, tout signe est susceptible de susciter l'apparition d'un nombre indéfini de signes-interprétants. Peirce appelle ce phénomène la "sémiose illimitée". Ainsi, si vous cherchez, par exemple, la définition d'un mot au dictionnaire (soit "occultation"), vous trouverez à la suite de ce terme un énoncé qui est censé vous en fournir le sens ("disparition passagère d'un astre par l'interposition d'un astre apparemment plus grand") mais qui lui aussi est formé de signes qui renvoient à d'autres définitions composées d'autres signes... et ainsi de suite ad infinitum.

Mais alors comment est-il possible d'attribuer un sens spécifique à un terme du lexique? Grâce au fonctionnement pragmatique du signe, répond Peirce. En effet, dire de la sémiose qu'elle renvoie sans cesse un signe à un autre, c'est décrire le signe sous un angle strictement sémiotique. Or, les signes font partie d'une praxis

1 C.S. PEIRCE, op. cit., 2.228, p. 121.

2 Cité par J. BuCHLER, Charles Peirce's Empirism, Kegan Paul, 1939, p. 114-115 et repris par G. DELEDalle, Théorie et pratique du signe, Paris, Payot, 1979, p. 33. 
communicationnelle où des interactants s'efforcent de partager leurs vues. Sous cet angle, l'interprétant peut être présenté comme l'effet momentané du signe sur l'esprit de l'interprète. "Occultation" est ainsi un terme du lexique qui ne prendra sens qu'au regard de son contexte d'apparition ("L'occultation de cette pièce est mal pensée..."). La sémiose illimitée est de la sorte suspendue le temps d'une interaction.

Résumons-nous: potentiellement, le signe doit pouvoir toujours renvoyer à un signe interprétant. Soulignons au passage la parenté existant entre cette conception et celle de Deleuze et Guattari: une langue va toujours d'un dire à un dire. Mais pragmatiquement la communication exige que ce processus soit enrayé (ne fût-ce que le temps de l'énonciation). La sémiosis illimitée du signe sera donc contrecarrée par une "force" qui aboutira à la figer et à la clôturer: c'est l'habitude. Comme le cours d'eau qui se creuse un lit, c'est en effet par un long et lent processus d'habituation, dira Peirce, que la définition d'un concept est (temporairement) établie. Le sens d'un signe n'est ainsi qu'un moment dans la dynamique des signes qui, elle, entretient toujours la sémiose.

Par cette présentation, Peirce cherche à nous montrer que les signes forment avant tout un système de relations dont la dynamique conduit à en définir les termes et non l'inverse. Si, d'un point de vue sémiotique, les signes sont des noeuds de relations - des relations de relations- d'un point de vue pragmatique, l'attribution du sens reste une opération fugace, dépendante d'habitudes acquises et sujette à révision.

Parallèlement à ses réflexions sur la dynamique triadique du signe (les relations representamen, objet et interprétant), Peirce a développé une "classification" du signe qui ne repose pas sur un découpage empirique des signes existants (le verbe, l'image, le geste,...) mais résulte d'une réflexion logico-phénoménologique (qu'il appelait "phanéroscopie"). Celle-ci le conduit à considérer dans l'ordre des phénomènes trois modes d'être: la priméité catégorie du sentiment et de la qualité, la secondéité catégorie du fait "brut" (c'està-dire non pensé) et la tiercéité catégorie de la relation pensée, de la loi. A chacune de ces catégories, Peirce associera notamment une opération sémiotique particulière -l'icône, l'indice et le symbole- en fonction du type de relation -similarité, contiguité, conventionnalitéétabli entre le signe et ce qu'il représente.

Ces opérations doivent maintenant être entendues par rapport au double mouvement du signe décrit ci-dessus. Si, sur le plan sémio- 
tique, l'icône, l'indice et le symbole représentent chacun un degré de complexité croissant du signe, sur le plan pragmatique, ces opérations s'organisent de telle sorte que les plus conventionnelles et arbitraires -les symboles- ne peuvent agir sans l'aide des plus naturelles et motivées -les icônes.

Ainsi, par exemple, dans un système parfait de notation logique, Peirce montrera que les symboles -qui sont les seuls signes "généraux" et "la généralité est essentielle au raisonnement"- ne peuvent se passer d'indices et d'icônes. Des indices parce que sans eux on ne pourrait distinguer le monde réel du monde de l'imagination. Des icônes parce que

\begin{abstract}
"avec ces deux genres de signes seuls [les symboles et les indices], on peut exprimer n'importe quelle proposition; mais on ne peut pas raisonner sur elle, car le raisonnement consiste dans cette observation que là où se trouvent certaines relations, il s'en trouve d'autres, et il requiert en conséquence que les relations raisonnées soient exprimées dans une icône"1.
\end{abstract}

Plus largement, Peirce montrera que l'icône est indispensable dans tout processus communicationnel: "la seule façon de communiquer directement une idée est par le moyen d'une icône; et toute méthode indirecte pour communiquer une idée doit dépendre pour son

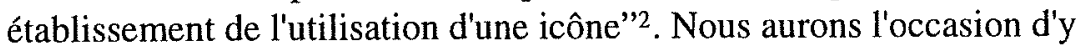
revenir.

\title{
Les trois processus iconiques: l'image, le diagramme et la métaphore
}

La métaphore étant une sorte d'icône, reste à préciser la nature de celle-ci. Pour Peirce, il s'agit d'un "signe qui renvoie à l'objet qu'il dénote simplement en vertu des caractères qu'il possède, que cet objet existe réellement ou non. (...). N'importe quoi, qualité, individu existant ou loi, est l'icône de quelque chose pourvu qu'il ressemble à cette

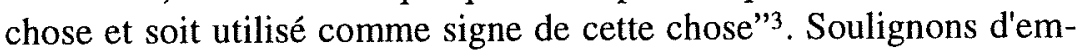
blée une double caractéristique: l'icône n'est pas par essence une représentation d'ordre matériel d'un objet (même si elle peut l'être à certains moments), pas plus qu'elle ne renvoie à une réalité matérielle qui lui serait extérieure (quoiqu'elle puisse le faire dans certains cas).

1 C.S. PEIRCE, op. cit., 3.363, p. 145-146.

2 Idem, 2.278, p. 149.

3 Idem, 2.247, p. 140. 
Par exemple, nous dit Peirce, "en contemplant un tableau, il y a un moment où nous perdons conscience qu'il n'est pas la chose, la distinction entre le réel et la copie disparaît, et c'est sur le moment un pur rêve -non une existence particulière et pourtant non générale. A ce moment nous contemplons une icône"1.

Métaphore, image et diagramme forment chacun un genre particulier d'icône. Comme les trois processus sémiotiques (icône, indice et symbole), chacun d'eux est rattaché à une catégorie "phanéroscopique": la catégorie du sentiment et de la qualité pour l'image, de la lutte et du fait brut pour le diagramme, de la pensée et de la loi pour la métaphore. Dès lors, je considère que ces icônes s'inscrivent elles aussi dans la dynamique sémio-pragmatique des signes. Ainsi la métaphore est, sur le plan sémiotique, une icône conventionnelle mais qui implique, pour être saisie, le recours à quelques diagrammes et à quelques images (naturelles). Abordons maintenant chaque type d'icône.

\section{L'image}

L'image forme, en quelque sorte, la ressemblance à l'état pur. C'est l'icônicité au sens strict, c'est-à-dire considérée indépendamment de sa matérialisation. Un tableau, une photo, un schéma, une métaphore sont bien sûr des images au sens où ils reposent tous fondamentalement sur un rapport de ressemblance potentiel. Mais la représentation mentale, le "mental model"', l'analogie, sont des images plus authentiques $^{3}$ dans la mesure où ils ne dépendent pas d'une (quelconque) matérialisation.

L'image -degré zéro de l'icône-est au cœur de la compréhension du signe, y compris de ceux qui reposent sur des processus arbitraires et conventionnels (les symboles). Sans icônes-images, il n'y a ni compréhension ni communication possibles. Ainsi, nous dit Peirce, un symbole comme le mot «aime» dans l'énoncé «Ezéchiel aime Houlda» ne peut être saisi que par le truchement de l'image: "l'effet du mot «aime» est que la paire d'objets dénotée par la paire d'indices

1 Idem, 3.362, p. 144-145.

2 P. Johnson-Laird., Mental models, Cambridge, Cambridge University Press, 1983.

3 Peirce oppose le terme "authentique" à celui de "dégénéré". Reprenant cette terminologie aux mathématiques, il veut par là indiquer que la relation triadique representamen, objet et interprétant met ou non en relation des objets de même nature phanéroscopique. 
Ezéchiel et Houlda est représentée par l'icône, ou l'image que nous avons dans l'esprit, d'un amoureux et de sa bien-aimée"1.

\section{Le diagramme et la "théorie des graphes existentiels"}

Les diagrammes consistent à représenter les relations entre des choses par des signes qui montrent les mêmes relations. Le diagramme est une icône de relations essentiellement dyadiques. Peirce en a proposé une opérationnalisation dans sa "théorie des graphes existentiels". Cette théorie avait pour ambition de proposer des formalismes graphiques pour tout problème qui était posé à la logique. Par "graphe" plus précisément Peirce entendait l'expression propositionnelle, dans le système des graphes existentiels, de tout état possible de l'univers du discours, c'est-à-dire du domaine des objets dont on va parler. Et il justifiait ce mode de représentation «diagrammatique» "par le fait que la nature et les habitudes de notre esprit nous le font saisir d'emblée, sans que nous ayons à faire l'effort de nous rappeler une règle sans rapport aucun avec nos modes d'expression habituels et naturels". Plus exactement, l'un des avantages des graphes est de conduire "plus directement à l'ultime analyse des problèmes logiques qu'aucune espèce d'algèbre jamais inventée"2. Parce que "le raisonnement par diagrammes est le seul raisonnement véritablement fécond" 3 .

Mais entre le monde de la logique et celui du langage verbal, il y a un rapport étroit. En effet, selon Peirce, «le langage n'est pas autre chose qu'une sorte d'algèbre». Or, "toute équation algébrique est une icône, dans la mesure où elle rend perceptible, par le moyen des signes algébriques (lesquels ne sont pas eux-mêmes des icônes), les relations existant entre les quantités visées". Plus précisément, il dira que «l'algèbre n'est pas autre chose qu'une sorte de diagramme». De sorte que le langage est fondamentalement de nature diagrammatique.

Jakobson a insisté sur ce fait dès 1966. Commentant Peirce, il tente de montrer que le langage n'est pas autre chose qu'une sorte de diagramme de relations. Partant de la proposition de Peirce selon laquelle "pour qu'une phrase puisse être comprise, il faut que l'arrangement des mots en son sein fonctionne en qualité d'icônes", il

1 C.S. PEIRCE, op. cit., 2.295, p. 163.

Idem, 3.619.

3 Idem, 4.571. 
avance que "si la chaîne de verbes veni, vidi, vici nous informe de l'ordre des actions de César, c'est d'abord et avant tout que la séquence des parfaits coordonnés est utilisée pour reproduire la succession des événements relatés. L'ordre temporel des procès d'énonciation tend à refléter l'ordre des procès d'énoncé, qu'il s'agisse d'un ordre dans la durée ou d'un ordre selon le rang. Une séquence comme «Le Président et le Ministre prirent part à la réunion» est beaucoup plus courante que la séquence inverse, parce que le choix du terme placé le premier dans la phrase reflète la différence de rang officiel entre les personnages." De même, il y aurait "un net caractère diagrammatique, non seulement dans la combinaison des mots en groupes syntactiques, mais aussi dans la combinaison des morphèmes en mots"1.

Les développements de la pragmatique linguistique constituent aussi, à mon sens, une façon de souligner la nature diagrammatique du langage. En effet, les pragmaticiens ont montré comment tout acte de langage situe un énoncé dans un contexte spatio-temporel déterminé (en attribuant une place aux interlocuteurs en présence, en situant l'énoncé dans un espace-temps déterminé, etc.). L'énoncé en garderait une certaine image sous la forme de traces linguistiques (des indices pour Peirce): pronoms personnels, démonstratifs, adverbes de lieux et de temps, modalités verbales, etc. "Le sens d'un énoncé, déclarait Ducrot, c'est, pour moi, une description, une représentation qu'il apporte de son énonciation, une image de l'événement historique constitué par l'apparition de l'énoncé". En quoi consiste cette image de l'énonciation inscrite dans l'énoncé? En ce que celui-ci "se présente comme produit par un locuteur, désigné en français (...) par le pronom et les différentes marques de la première personne" et "comme adressé par le locuteur à un allocutaire ... désigné... par les pronoms et les marques de la deuxième personne (...)"2. Ces marques linguistiques (pronoms, adverbe, etc.) organisées à l'intérieur de l'énoncé forment ainsi une sorte de diagramme des relations que l'énonciation tente d'établir.

C'est en tous cas en ce sens que je comprends l'interprétation avancée par Peirce à propos de l'énoncé «Ezéchiel aime Houlda». Celui-ci n'est pas, au sens strict, une icône mais bien un diagramme de relation. En effet, pour que cette phrase produise du sens, il faut

1 R. JAKOBSON, A la recherche de l'essence du langage, in E. BENVENISTE, A. Martinet, I. Fonagy, R. Jakobson et al., Problèmes du langage, Paris, Gallimard, coll. Diogène, 1963, p. 23-38.

2 O. DUCRoT, Les mots du discours, Paris, Éd. de Minuit, 1980, p. 34-35. 
qu'elle contienne des marques linguistiques capables de désigner les personnes impliquées par elle. A cet égard les signes symboliques ne sont d'aucune utilité car ce sont des signes généraux et descriptifs et "aucune description ne permet de distinguer le monde réel du monde de l'imagination. D'où le besoin de pronoms et d'indices (...)"'. "Ezéchiel et Houlda doivent donc être ou contenir des indices; car sans indices il est impossible de désigner ce dont on parle. Une description superficielle ne préciserait pas si ce sont simplement des personnages d'une ballade; mais qu'ils le soient ou non, les indices peuvent les désigner. Or, l'effet du mot «aime» est que la paire d'objets dénotée par la paire d'indices Ezéchiel et Houlda est représentée par l'icône, ou l'image que nous avons dans l'esprit, d'un amoureux et de sa bien-aimée"2. C'est donc bien l'organisation diagrammatique (sorte d'équation algébrique de l'amour existant entre Ezéchiel et Houlda) de l'énoncé -deux indices reliés par un symbole-qui a pour effet de produire une icône (une image) dans l'esprit du récepteur. Le langage, nous disait Deleuze, est une carte et non un calque...

\section{La métaphore}

Par rapport au diagramme, la métaphore présente un degré de conventionnalité supplémentaire: elle consiste non plus à illustrer des relations existant entre des choses ou entre des parties de chose mais bien à représenter une relation sémiotique par une relation sémiotique similaire.

Contrairement à la rhétorique classique, Peirce nous présente la métaphore comme un processus iconique. La métaphore verbale constitue donc un cas particulier qui ne peut servir de référence pour une définition générale. Dans la rhétorique verbale, la métaphore est d'ordinaire présentée comme ne disposant pas d'outil comparatif ( $« \mathrm{Ce}$ garçon a une agilité de singe» vs «Ce garçon est agile comme un singe»). Dans la mesure où les deux termes pivots, le comparant (singe) et le comparé (garçon), sont l'un et l'autre indiqués, ou bien que seul le comparant est marqué dans le discours («Un vrai singe parut alors sous mes yeux»), les rhétoriciens parlent respectivement de métaphores in praesentia et in absentia ${ }^{3}$.

1 C.S. PEIRCE, op. cit., 3.363; p. 145.

2 C.S. PEIRCE, op. cil., 2.295 ; p. 163.

3 Cf. G. Molinié, Dictionnaire de rhétorique, Paris, Librairie Générale Française, 1992, p. 213-216. 
Dans l'image concrète (photo, dessin,...), l'absence de marqueur comparatif et la quasi-impossibilité de mentionner la qualité attribuée (l'agilité du singe) n'autorisent ni les comparaisons ni les métaphores in absentiae. Le comparant et le comparé sont co-présents et ont tendance à se confondre dans l'esprit du spectateur. Ainsi dire de quelqu'un que «C'est un singe» peut être exprimé graphiquement par un singe se comportant comme un être humain. Mais dans ce dernier cas, le lecteur de l'image voit simultanément le singe et l'être humain. Quant à la qualité attribuée -l'agilité- elle n'est pas "lue" par le spectateur mais inférée par lui, si bien que d'autres rapprochements peuvent être réalisés: l'aspect simiesque, malin ou encore imitateur de l'être en question. C'est cette capacité de (con)fusion de signes qui est fondamentale dans la métaphore. Toutefois, cette confusion n'est pas totale. En effet, il subsiste toujours le sentiment -même vague- d'une dualité, d'une opposition entre deux termes incongrus (l'homme vs le singe). C'est en cela qu'on peut dire de la métaphore qu'elle implique une dimension diagrammatique. Sans elle, rien ne permettrait de distinguer la métaphore de l'icône-image.

Si cette dernière représente quelque chose par quelque chose d'autre qui lui ressemble, la métaphore quant à elle ne constate pas une ressemblance. Au contraire, elle produit des relations de similitude, elle construit des images. En fait elle met en rapport dynamique/diagrammatique deux univers distincts en soulignant les traits qu'ils ont/auraient en commun. De ce point de vue, on peut parler de la nature conventionnelle de la métaphore (elle provient d'associations favorisées par la culture, la vie sociale, les modes de représentations, ...).

Mais la métaphore est une icône et à ce titre, elle se donne à percevoir comme n'importe quelle image. La verbalisation d'une icône métaphorique va alors engendrer un second degré de conventionnalité: le mot -le symbole - va venir fixer l'association produite au niveau de l'icône dans un signe substitutif: c'est la métaphore in absentiae. Le risque alors est de voir la conventionnalité des associations l'emporter sur la force heuristique de l'icône. L'agilité représentée par le concept de singe, la vieillesse par celle du "soir" de la vie, etc. deviennent ainsi des métaphores éculées et hypercodifiées. Cependant, même dans ces cas extrêmes, la métaphore reste une icône et, par conséquent, demeure capable de favoriser de nouvelles interprétations (cf. ci-dessous le paragraphe "métaphore et cognition"). 
Là où le diagramme tente de simuler des relations dyadiques par la mise en relation de signes qui sont de nature foncièrement différentes (un simulateur informatique des mécanismes de la vision, par exemple, diffère en nature du fonctionnement neurophysiologique du système oculaire), la métaphore, elle, amalgame ces univers dans un signe unique (en parlant par exemple de notre "processeur visuel" ou en montrant une découpe du cerveau laissant entrevoir un ordinateur relié au nerf optique, etc.). En ce sens, elle favorise les rapprochements sémantiques mais en autorisant aussi l'indifférenciation des unités et la confusion des niveaux.

\section{Présentation diagrammatique}

Afin de visualiser les relations établies entre les différents genres d'icônes, je me propose de les présenter ci-dessous sous forme diagrammatique, suivant en cela les directives de Peirce en matière de notation logique.

Soit

$X, Y, Z$
$x, y, z$
-
$=$
$\leftarrow$
$\leftarrow$

des signes

des référents, des "états de choses"

la relation dyadique

la relation de ressemblance

la relation de représentation

la relation de représentation par ressemblance

Alors: $\quad$ X est une icône si

$$
\mathbf{X}=\mathbf{Y}
$$

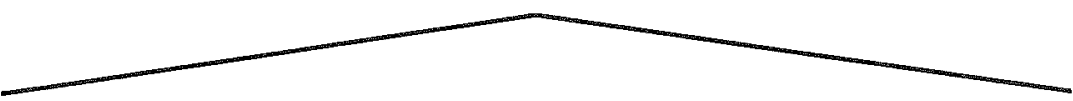

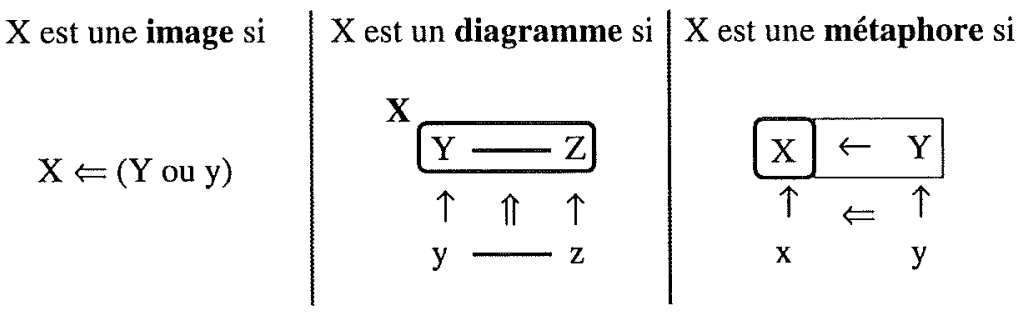




\title{
3. Métaphores et cognition
}

D'après Peirce, on ne pourrait donc pas se passer d'icônes, c'està-dire que même les signes les plus arbitraires et conventionnels -les symboles-nécessitent le recours à des images, des diagrammes et/ou des métaphores. Mais ces dernières occuperaient-elles une place privilégiée dans le monde des icônes?

\begin{abstract}
"La plupart des gens ont besoin d'exemples et de métaphores pour comprendre une proposition ou un concept abstraits, les seules définitions suffisent rarement. A-t-on jamais tiré toutes les conséquences, sur le plan cognitif, de ce fait massivement observable? Avec Lakoff et Johnson, nous faisons ici l'hypothèse que les êtres humains ne peuvent véritablement comprendre des concepts et des propositions abstraits qu'en les assimilant métaphoriquement, ou par association, à des expériences concrètes, qu'elles soient émotionnelles, physiques, sensori-motrices, spatiales, sociales, etc. Derechef, nous ne comprenons que si, à une extrémité ou à une autre de nos réseaux associatifs et de nos activités élaboratrices, se découvre quelque icône (qui n'est pas nécessairement une image)"'1.
\end{abstract}

Nous souscrivons bien entendu à cette dernière phrase (qui exploite d'ailleurs explicitement la terminologie de Peirce). Mais peuton suivre aussi P. Levy quant à la place prioritaire qu'il semble accorder, au sein des processus cognitifs, à la métaphore?

Peirce a longuement traité de ces mécanismes cognitifs au travers de ses réflexions logiques et sémiotiques, principalement à partir de la notion -centrale dans son système- d'interprétant. Un signe, disait Peirce, crée dans l'esprit de la personne à laquelle il s'adresse un signe équivalent ou peut-être plus développé qu'il appelle l'interprétant du premier signe. Le processus d'interprétance tente de décrire cet effet du signe sur l'esprit de l'interprète. Les icônes y jouent un rôle déterminant. Sans entrer ici dans une discussion approfondie de cette notion ${ }^{2}$, je voudrais m'arrêter sur un des processus qui y est à l'œuvre et qui retient d'ordinaire l'attention des exégètes de Peirce: le mécanisme d'inférence.

1 P. LÉVy, L'idéographie dynamique. Vers une imagination artificielle?, Paris, Éd. La découverte, 1991, p. 97-98.

2 J'en ai longuement discuté in Signe et autoréférence. Essai de modélisation de la communication sur base de la théorie sémio-pragmatique de Charles $S$. Peirce, Thèse doctorale, Louvain-la-Neuve, Département de communication, UCL, 1988. 


\section{Les trois modes d'inférence}

Peirce s'est beaucoup intéressé aux mécanismes d'inférence dans le cadre notamment de ses réflexions sur le syllogisme. Il distinguait trois sortes d'inférence: la déduction, l'induction et l'abduction. S'inspirant de la terminologie kantienne, Peirce nous dit de la première qu'elle est analytique tandis que les deux autres sont synthétiques et il représente ces relations de la façon suivante':

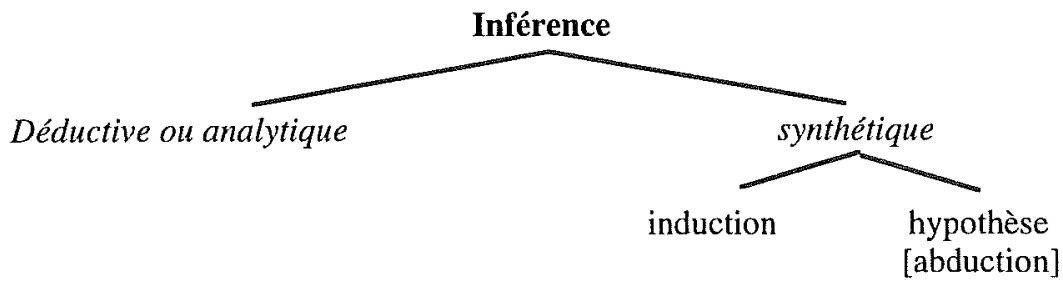

La déduction consiste à tirer une conclusion (un "résultat") à partir de l'application d'un principe général (une "règle") à un cas particulier. Peirce prend l'exemple suivant:

$$
\begin{array}{ll}
\text { Règle } & \text { - tous les haricots de ce sac sont blancs } \\
\text { Cas } & \text { - ces haricots proviennent de ce sac } \\
\text { Résultat } & \text { - ces haricots sont blancs }
\end{array}
$$

Dans ce type d'inférence la conclusion est contenue virtuellement dans les prémisses et est donc simplement déduite par analyse de celles-ci. Mais même dans ce mode de raisonnement élémentaire, Peirce considère que des mécanismes iconiques sont nécessairement mis en jeu: "tout raisonnement déductif, même le simple syllogisme, implique un élément d'observation. La déduction consiste en effet à construire une icône ou un diagramme dont les relations des parties doivent présenter une complète analogie avec celles des parties de l'objet du raisonnement, de l'expérimentation sur cette image dans l'imagination et de l'observation du résultat de façon à découvrir des relations qu'on n'avait pas remarquées et qui étaient cachées dans les parties. Prenons, par exemple, la formule syllogistique

$$
\begin{aligned}
& \text { Tout } M \text { est } P \\
& S \text { est } M \\
& \therefore S \text { est } P
\end{aligned}
$$

Elle est réellement un diagramme des relations de $S, M$ et $P$. Le fait que le moyen terme se trouve dans les deux prémisses apparaît

1 Cf. C.S. PEIRCE, op. cit., 2.623. 
réellement et cela est nécessaire sans quoi la notation n'aurait aucune valeur"l.

P.Lévy souligne à juste titre combien cette présentation est proche de la conception de Johnson-Laird. "Là où le psychologue invoque des modèles mentaux, le philosophe fait appel à des icônes. (...) Selon Peirce, tout raisonnement met en jeu l'inspection d'icônes (ce que l'on pourrait traduire par: la simulation de modèles men$\operatorname{tau} x)^{\prime \prime 2}$. En effet, nous dit Peirce, "le raisonnement consiste dans cette observation que là où se trouvent certaines relations, il s'en trouve d'autres, et il requiert en conséquence que les relations raisonnées soient exprimées dans une icône"3.

Toutefois, les types d'icônes mentionnées ici sont de l'ordre des images et des diagrammes mais non des métaphores. On le comprend aisément puisqu'il s'agit de découvrir des rapports internes aux prémisses. Qu'en est-il maintenant des inférences dites "synthétiques"?

L'induction s'attache à inférer une règle à partir de l'observation d'un cas particulier et d'un résultat. Il s'agit cette fois d'un raisonnement qui généralise à une classe ce qui est vrai d'un certain nombre de cas appartenant à cette classe. L'induction, à l'opposé de la déduction, représente une connaissance nouvelle qui n'est pas inférable analytiquement à partir de la connaissance de ce qui est vrai pour le cas. Son intérêt est d'établir des connaissances générales, des règles qui serviront ensuite de prémisses au raisonnement déductif.

L'abduction apporte également de nouvelles connaissances mais de nature différente de celles produites par l'induction. Elle consiste à inférer un cas à partir d'une règle et d'un résultat. "Supposons -écrit Peirce-que j'entre dans une pièce et que j'y trouve un certain nombre de sacs qui contiennent différentes sortes de haricots. Sur la table, il y a une poignée de haricots blancs; et, après une petite recherche, je trouve que l'un des sacs contient seulement des haricots blancs. J'en infère tout de suite que, probablement ou très vraisemblablement, cette poignée de haricots blancs provient de ce sac. (...)

\footnotetext{
1 Idem, 3.363, p. 146.

2 P. LÉVY, op. cit., p. 92.

3 C.S. PEIRCE, op. cit., 3.363, p. 145.
} 
Règle - tous les haricots de ce sac sont blancs.

Résultat - ces haricots sont blancs.

Cas - ces haricots proviennent [probablement] de ce sac"1.

Cette fois, l'inférence aboutit à la production d'énoncés conjecturaux et hypothétiques. Il ne s'agit plus, comme c'était le cas le cas pour l'induction, de produire des règles générales mais plutôt d'avancer des hypothèses permettant de relier les deux prémisses. Comme le souligne Carontini, c'est là précisément que se situe la faiblesse du raisonnement abductif: entre le résultat et la règle la relation établie ne peut être que de nature hypothétique et conjecturale. Mais cela est compensé par sa capacité à opérer, grâce à des choix interprétatifs parfois très audacieux, de véritables sauts cognitifs pouvant conduire à des connaissances nouvelles ${ }^{2}$. La présomption, ou plus exactement, l'abduction, dit Peirce, est "le seul genre de raisonnement qui fournit de nouvelles idées, le seul genre qui soit, en ce sens, synthétique. (...) Sa seule justification est que cette méthode est la seule voie dans laquelle il peut y avoir quelque espoir d'arriver à une explication rationnelle"3.

Abduction, déduction et induction forment, dans la conception de Peirce, les trois modalités fondamentales de tout raisonnement:

elles "participent au processus unifié de la connaissance en y apportant une contribution spécifique. Face à des données problématiques le rôle de l'abduction est d'émettre une hypothèse explicative. C'est le moment proprement créatif, originaire de la connaissance, dont la fécondité et le bien-fondé restent à justifier. Le rôle de justification et de contrôle de l'abduction est assumé par l'inférence déductive et par l'inférence inductive. Par une exploration analytique de l'hypothèse, l'inférence déductive doit permettre de sélectionner parmi les multiples conséquences possibles de l'hypothèse, les conséquences qui pourraient être pertinentes pour la corroboration de celle-ci (...). Il reviendra à l'induction de procéder à une vérification des "prédictions virtuelles" proposées par la déduction; toute vérification de ces prédictions rendra l'hypothèse plus plau-

1 Idem, 2.623

2 E. CARONTINI, Inférence et encyclopédie, notes ronéotypées, Montréal, 1988, p. 20. Une partie de celles-ci a été publiée sous le titre "Le rôle de l'abduction dans le processus d'interprétation" in Technologies et symboliques de la communication, Colloque de Cerisy sous la direction de L. SFEz et G. CouTLÉE, Grenoble, PUG, 1990, p. 216-227.

3 C.S. PEIRCE, op. cit., 2.776-777. 
sible et permettra au processus de la connaissance d'accomplir une étape ultérieure"!

$\mathrm{Si}$, pour Carontini, ces trois processus inférentiels forment système (l'un appelant nécessairement l'autre), pour Levy l'abduction doit être mise en avant:

"Le raisonnement abductif consiste à faire fonctionner un modèle mental de l'ensemble d'une situation qui soit compatible avec les parties de la situation que l'on peut observer réellement. Il s'agit de reconstruire l'ensemble d'une histoire à partir de fragments connus. (...) L'abduction ne va pas sans un va-et-vient permanent entre la construction et la simulation de modèles mentaux, d'une part, et l'observation en vraie grandeur, d'autre part. Nous faisons l'hypothèse que la déduction et l'induction ne sont que des cas particuliers ou des éléments partiels d'une activité de raisonnement "complète" définie comme construction, simulation et comparaison de modèles mentaux."2.

\section{Abduction et métaphore}

Dans ces deux conceptions, l'abduction joue donc un rôle déterminant. Ci-dessus, nous avons vu que l'originalité de l'inférence abductive consistait à établir une relation hypothétique entre une donnée à expliquer (le "résultat") et un principe explicateur (la "règle"). Carontini, se basant sur différents travaux ${ }^{3}$, fait remarquer que le degré de créativité de l'abduction dépend de la qualité de cette relation (il nomme cette dernière "loi-médiation"). Plusieurs degrés sont ainsi distingués dans l'activité abductive en fonction du type de loi-médiation à laquelle l'inférence a recours. Je les ramènerai à deux grands cas de figure.

Soit la règle nécessaire à l'inférence du cas à partir du résultat existe comme telle dans l'encyclopédie de l'interprète. Ici, l'abduction repose tantôt sur l'application semi-automatique d'une règle (le plus simple décodage présuppose ce type d'inférence: par exemple "reconnaître" malgré les inévitables variations de timbre, de hauteur, etc. les phonèmes utilisés par le locuteur et répertoriés dans le code

1 E. CARontini, op. cit, p. 27.

2 P. LÉVY, op. cit., p. 92-93.

3 M. BonfantinI, "Abduction a priori, brain: for a Research program", Versus, n³4, 1984; U. ECO, "Guessing; from Aristote to Sherlock Holmes", Versus, n³0, 1981; M. FERRARESI, "Le abduzioni nel testo", Versus, Quademi di studi semiotici, n³4, 1983; G. PronI, "Genesi e senso dell'abduzione in Peirce", Versus, n²8, 1981. 
linguistique partagé par les interlocuteurs), tantôt la règle est trouvée par sélection dans le domaine de l'encyclopédie disponible (cette fois, l'interprète doit deviner parmi les différentes lois disponibles celles qui sont les plus adaptées à expliquer le résultat: par exemple, dans le cas de l'enquête policière, se demander s'il s'agit d'un crime passionnel, d'un règlement de compte, d'un scandale politique,...).

Soit nous avons affaire à un type d'inférence dans laquelle la loimédiation exige une certaine forme de créativité et d'invention. En suivant les suggestions de Bonfantini, Carontini distingue ici deux sous-types: dans le premier, "la loi médiation serait une sorte d'extension à un champ sémantique nouveau d'un principe explicateur qui appartient à un champ relativement éloigné dans l'encyclopédie: la métaphore constitue l'exemple typique de cette forme d'abduction. Toute métaphore authentique suppose que l'interprète soit capable d'opérer des transferts, qu'il soit capable de déplacer un principe d'explication d'un champ sémantique à un autre. La métaphore est une opération risquée, éminemment faillible et profondément conjecturale, mais qui ne rend pas impossible la communication. Il y a dans toute métaphore à la fois quelque chose de profondément connu et d'éminemment nouveau. La métaphore assure, permet, fonde la communication et, en même temps, elle lui offre la possibilité d'explorer des territoires inconnus"1.

Dans le second sous-type, "la loi médiation est inventée ex novo; elle constitue donc quelque chose de radicalement nouveau par rapport à l'univers du savoir du sujet connaissant" 2 . Celle-ci ne se rencontre guère en dehors de l'acte de connaissance scientifique car, dans le domaine de la communication, quelle que soit l'innovation, "il faut que, d'une façon ou d'une autre, cette innovation, cette créativité, puisse être maintenue dans les limites d'un système sémantique global, partiellement ou totalement commun aux sujets communicants"3. Cette dernière remarque appelle, me semble-t-il, une réserve: peut-on isoler l'acte de connaissance scientifique du système sémantique commun aux sujets communicants (ici les scientifiques)? Peut-on, sur cette base, distinguer discours ordinaire et discours scientifique? Ces questions reviennent à se demander si la création ex novo, la nouveauté absolue, serait possible.

1 E. Carontini, op. cit., p. 38-39.

2 Idem, p. 40.

3 Ibidem. 


\section{La métaphore dans l'acquisition et la transmission de connaissances nouvelles}

La discussion de la notion d'abduction nous conduit à nous interroger maintenant sur le rôle de la métaphore dans l'acquisition de connaissances. Il est vrai, comme le souligne Eco, que "la métaphorologie moderne -c'est là un de ses traits marquants-a insisté davantage sur le rapport entre métaphore et découverte scientifique, et, plus généralement, entre métaphore et connaissance plutôt que sur le rapport entre métaphore et poésie"'. Cela provient sans doute du pouvoir heuristique qu'on lui prête. Mais, au sens strict, la métaphore est-elle un outil de découverte? Et si c'est le cas, à quelles conditions le seraitelle?

\section{Invention vs découverte scientifique}

Dans le système de pensée qu'il s'est construit, Peirce a défini la condition sémiotique de la nouveauté. Parlant de l'objet du signe, il déclare: "le signe ne peut que représenter l'objet et en dire quelque chose. Il ne peut ni faire connaître ni reconnaître l'objet; car c'est ce que veut dire dans le présent volume objet d'un signe; à savoir ce dont la connaissance est présupposée pour pouvoir communiquer des informations supplémentaires le concernant"2. Pour qu'il y ait acquisition d'information, il faut que celle-ci ait un rapport (direct ou indirect peu importe) avec quelque chose que l'interprète connaît déjà. La création ex novo -si elle existe- doit dès lors s'entendre dans ce cadre, c'est-à-dire celui où "toute connaissance est déterminée logiquement par des connaissances antérieures"3.

Mais comment germerait cette nouveauté relative? Pour répondre à cette question, revenons au processus d'interprétance. Au sein de ce dernier, le renvoi d'un signe à un autre peut résulter soit de la reconnaissance d'une relation de similarité entre les deux signes (l'icône), soit de l'établissement d'une relation dynamique -de contiguité contextuelle- entre eux (l'indice), soit encore de l'association d'idées générales (le symbole). Mais l'icône présente un avantage sur les deux autres procédés: “(...) une des grandes propriétés distinctives de l'icône est que par son observation directe peuvent être découvertes

1 U. ECO, Les limites de l'interprétation, Paris, Grasset, 1992, p. 168.

2 C.S. PeIRCE, op. cit., 2.231, p. 123, je souligne.

3 Idem, 5.265. 
concernant son objet d'autres vérités que celles qui suffisent à déterminer sa construction. Ainsi, au moyen de deux photographies on peut tracer une carte, etc."'.

Des trois genres de signes, seule l'icône serait capable de favoriser l'émergence stable d'un nouveau signe, émergence non inférable à partir de ses prémisses. En propre dans toute icône -donc a fortiori dans toute métaphore- il y a à la fois "quelque chose de profondément connu et d'éminemment nouveau". C'est cela qui fait de ce signe le fondement (la "priméité" devrions-nous dire) du système sémiotique peircien. Ainsi, on ne sort jamais de l'univers des signes: la nouveauté n'est pas dans le dévoilement d'une nouvelle réalité ni dans la "découverte" mais plutôt dans l'établissement de nouvelles relations parmi des relations connues, dans la création de nouveaux signes inférés (abduits) de signes connus, nouvelles relations/nouveaux signes demandant à être légitimés par l'observation, l'expérimentation ou tout simplement par le système communicationnel en place.

\section{Le double processus métaphorique dans les discours de vulgarisation scientifique}

Si le discours vise maintenant à transmettre des informations scientifiques, la métaphore remplit-elle la même fonction heuristique? Je me limiterai ici à quelques remarques sur un cas particulier de communication des connaissances: la transmission à un public non expert de connaissances "établies", celle-ci exploitant tant et plus les procédés métaphoriques. La raison à cela tient probablement au fait que la métaphore réussit le tour de force de proposer à son destinataire, sous la forme d'une représentation unique (verbale ou iconique), une vue synthétique de données scientifiques, un modèle cognitif analogique et un mode de relation convivial (où l'humour, le clin d'œil, la complicité peuvent rivaliser). Toutefois, toute médaille à son revers. En l'occurrence, la métaphore ne rend compte que de façon approximative des données scientifiques (nous verrons qu'elle est incapable d'identifier les termes qu'elle met en relation); elle ne propose qu'une vision des choses -fût-elle originale- et enfin, par la participation du destinataire qu'elle sollicite, elle ne favorise pas la prise de distance critique.

C'est pour de telles raisons, me semble-t-il, que Bachelard a mis en garde contre de tels procédés. Dans un essai sur la "psychanalyse

1 Idem, 2.279, p. 150. 
de la connaissance objective", il s'emploie à en montrer le caractère préscientifique: "le danger des métaphores immédiates pour la formation de l'esprit scientifique, c'est qu'elles ne sont pas toujours des images qui passent; elles poussent à une pensée autonome; elles ten-

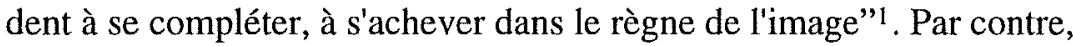
la science une fois constituée, la métaphore permettrait d'éclaircir des idées abstraites, d'illustrer un modèle théorique mais non de penser (au sens scientifique) l'expérience. Selon qu'elle se situe en amont ou en aval de la découverte scientifique, la métaphore serait perçue tantôt comme enfermante et sclérosante, tantôt comme illustrative et créatrice.

Qu'en est-il dans les discours de vulgarisation scientifique? Ceux-ci ont pour objet de reformuler des énoncés scientifiques à l'intention de publics non experts. Authier a constaté que cette reformulation -censée produire un équivalent du discours source- est couramment mise en scène dans les discours de vulgarisation: ainsi, le travail de reformulation se donne-t-il à voir sous la forme, par exemple, d'une communication imaginaire entre différents laboratoires scientifiques et un public non expert représenté, dans le document vidéo, par un "candide". Cette activité de mise en scène semble nécessaire à la légitimation du discours de vulgarisation dans la mesure où celui-ci ne tire sa valeur que du discours scientifique qui le fonde. Formellement, la vulgarisation se constitue donc autour d'une métaphore, celle de 1' "image en discours du dialogue rompu entre communauté scientifique et public"2. "Un «je parle pour d'autres» pourrait être la formule sur laquelle s'articule cette rhétorique de la médiation. (...) le vulgarisateur parle pour -à la place de l'un, scientifique- et parle pour -à l'intention de l'autre, public- avec les mots des deux, donc, dans un discours marqué par cette double détermination".

Par conséquent, dans la vulgarisation scientifique, un double processus métaphorique se développe. L'un, agissant au niveau de l'énonciation, porte sur la mise en place d'un dispositif de mise en scène de la communication -sorte de mise en expression de la métaphore du "dialogue rompu"; l'autre, agissant au niveau de la reformu-

1 G. BACHELARD, La formation de l'esprit scientifique, Paris, Vrin, $14^{\circ}$ édition, 1989 , p. 81 .

2 J. AuthiER, "La mise en scène de la communication dans des discours de vulgarisation scientifique", in Langue française, ${ }^{\circ} 53,1982$, p. 43.

3 Idem, p. 44. 
lation du contenu, s'attache à produire au sein de l'espace construit par le premier des métaphores du discours scientifique. Toute la question alors serait de pouvoir apprécier l'effet de ces métaphores "en abyme". Pour Authier, la mise en scène de la communication serait pour ces discours une manière de se donner, à l'instar des discours pédagogiques, un contexte institutionnel qui leur fait défaut.

Bien pensé, ce mode d'expression pourrait, il me semble, construire ce savoir sur le savoir de l'autre, ce "savoir décalé" dont parle Roqueplo: "Le boulanger est au four; il connaît la température, l'humidité, disons qu'il «sait» faire du pain. La boulangère, elle, est au magasin; elle connaît sa clientèle et elle «sait» compter ses sous. La boulangère sait reconnaître du bon pain, mais elle ne «sait» pas le faire et il est fort possible que, de son côté, le boulanger ne «sache» pas compter les sous. Pourtant la boulangerie fonctionne. Ils savent travailler ensemble, ce qui implique que chacun sache quelque chose sur le savoir de l'autre: en première approximation, c'est ce savoir sur le savoir de l'autre que j'appellerai un «savoir décalé»"'. En fait, il est sans doute illusoire de croire en la possibilité d'une diffusion scientifique large qui respecterait la structure intrinsèque des savoirs d'origine. Mieux vaut peut-être juger de la communication scientifique "à partir des décalages qu'elle institue, c'est-à-dire, en dernier ressort, à partir de ce qu'elle autorise, tant dans le domaine de l'acquisition (ultérieure) de connaissances que dans celui des pratiques concrètes que... cette communication doit aider à rendre possibles"2. Le double travail métaphorique de la vulgarisation, tout en rendant possible ce savoir décalé, en constitue sans doute aussi le reflet inscrit en filigrane dans le document de vulgarisation.

\section{Conclusions}

A partir des propositions de Peirce, nous pouvons maintenant décrire le fonctionnement métaphorique comme articulé autour d'un triple point de vue.

$1 \mathrm{Ph}$. Roqueplo, "Le savoir décalé," in Technologies et symboliques de la communication, op. cit., p. 75.

2 Idem, p. 80. 
Sur le plan sémiotique, la métaphore est une sorte d'icône. A la différence des images -et dans une moindre mesure des diagrammeselle ne propose pas un signe qui constate une ressemblance mais construit, sur la base de conventions (culturelles, sociales, scientifiques,...) une image syncrétique de deux champs sémantiques distincts en soulignant les traits qu'ils ont en commun.

A l'intérieur du système des signes, la métaphore joue un rôle de "broyeur" de concepts: elle présente sous la forme d'un signe unique deux concepts distincts ${ }^{1}$.

$\mathrm{Vu}$ la place qu'elle occupe au sein des processus iconiques, la métaphore est un signe mixte -conventionnel et naturel-donnant à voir la relation existant entre des choses qu'elle ne peut elle-même identifier. Elle est à la fois un signe analogique -elle se donne à voir comme une icône- et un signe digital -elle se constitue à partir de conventions sociales et culturelles. Dès lors, la verbalisation d'une métaphore attribue à celle-ci un second degré de conventionnalité: le mot, en nommant l'icône métaphorique, peut alors remplacer le représentant. Autrement dit, la copie -la métaphore verbale- peut se substituer à l'original sans en garder la trace: c'est la métaphore in absentiae.

Sur le plan pragmatique et communicationnel, l'organisation sémiotique des signes se renverse. Le signe symbolique, qui est parvenu -à force de conventions et d'arbitraires- à s'abstraire au maximum des contingences de l'univers de son représenté, a cette fois besoin d'indices et d'icônes pour être communiqué.

Au sein de l'icône, il en va de même: la métaphore implique toujours le recours à quelques diagrammes et images. Ainsi, comprendre une métaphore c'est nécessairement saisir qu'elle résulte de la confrontation dynamique de deux signes (aspect diagrammatique) -et ce, même si le comparant seul nous est donné à percevoir. D'autre part, c'est saisir -ou mieux abduire- le rapport d'analogie (aspect "image") qui relie ces signes et justifie leur rapprochement.

En définitive, cela revient à dire que pour communiquer quelque chose à quelqu'un, il faut que, directement ou indirectement, cela pro-

1 Ces concepts, éventuellement, se situent à des niveaux logiques différents et tendent alors à dissoudre les niveaux métalinguistiques (cf., ci-dessous, le "syllogisme en herbe" de Bateson). 
duise chez ce dernier quelque analogie ou image. Pour cette raison, on peut dire de la métaphore qu'elle remplit une fonction heuristique:

"La fonction des modèles analogiques n'est pas directement cognitive mais avant tout heuristique: elle est de donner à voir et de donner à dire, d'étendre et d'enrichir l'espace traitable"'.

Mais, ce faisant, nous voilà déjà introduit à la dimension cognitive des processus métaphoriques. Sur ce plan, la métaphore est étroitement liée à l'abduction. C'est elle qui propose, en un signe unique, de transférer un principe explicateur d'un champ sémantique à un autre. En ce sens, elle constitue l'outil idéal pour construire des hypothèses.

Pour marquer cette fonction cognitive de la métaphore, Bateson a proposé de donner de celle-ci une présentation syllogistique générale. Prenant pour modèle le célèbre "syllogisme Barbara", il écrit:

L'herbe est mortelle

Or les hommes sont mortels

Donc les hommes sont de l'herbe

Peu importe, déclare Bateson, que ce genre de raisonnement -qu'il nomme "syllogisme en herbe"- soit désapprouvé par les professeurs de logique classique: "il serait stupide de partir en guerre contre tous les syllogismes «en herbe», parce qu'ils sont l'étoffe dont l'histoire naturelle est faite. (...) L'ensemble du comportement animal, celui des répétitions anatomiques ou celui de l'évolution biologique -chacun de ces vastes domaines est ordonné et unifié par un entrelacs de syllogismes en herbe, que cela plaise ou non aux logiciens"2.

Mais, sur un autre plan, cela montre encore une fois que la métaphore n'est pas le propre des systèmes symboliques supérieurs (verbaux notamment). Bateson nous en donne la raison: "pour élaborer des syllogismes Barbara, il faut avoir préalablement identifié des classes, afin que les sujets et les prédicats puissent être différenciés. Mais, en dehors du langage, il n'y a ni classes nommées ni relations entre sujet et prédicat. Par conséquent, les syllogismes en herbe doi-

1 J. SChlanger, "La pensée inventive," in I. STEngers et J. SChlanger, Les concepts scientifiques, Paris, La découverte, 1989, p. 74-75.

2 G. BAtEson, La peur des anges. Vers une épistémologie du sacré, Paris, Seuil, 1989, p. 44. 
vent constituer le mode dominant d'interrelation communicationnelle des idées dans tous les domaines préverbaux"1.

Cela implique donc que l'on peut difficilement se passer du langage symbolique. La fonction cognitive de la métaphore accoucherait d'une souris si, à un moment ou à un autre, un langage symbolique ne venait identifier des classes, des sujets, des prédicats, etc. En ce sens, la création, envisagée par Lévy, d'une "idéographie dynamique" -sorte de simulateur informatique de modèles mentauxnous paraît théoriquement difficile à concevoir si, comme le propose l'auteur, elle ne faisait aucunement appel aux symboles².

Concrètement maintenant, qu'en est-il du "bon usage" de la métaphore? Pour répondre à cette question, procédons a contrario et examinons un exemple-type. Dans un article consacré à la vulgarisation de la physique des particules, l'auteur nous introduit au sujet en ces termes: "Le zoo des particules. De Newton à Hawking, les physiciens rêvent d'offrir aux hommes une grille de lecture de l'univers. Derrière les barreaux de la grille, une foule de particules s'agite. Rédiger les bonnes étiquettes sur les cages du zoo, aller dénicher dans la jungle universelle les espèces rares: la physique toucherait-elle au but?"3.

Plusieurs métaphores sont, dans cet extrait, exploitées: le zoo, la grille (de lecture), une foule (de particules), la jungle (universelle). Ces métaphores se renvoient l'une à l'autre par métonymie (la grille de la cage du zoo), par antithèse (ordre (zoo)/désordre (jungle), par équivalence (foule est assimilé ici à jungle), etc. Notons enfin que le terme grille est utilisé comme élément de la métaphore du zoo (les «barreaux de la grille») et permet le transfert à la métaphore du déchiffrement (par analogie à une ancienne acception où grille signifiait un "carton à jours conventionnels pour la lecture des textes rédigés en langage chiffré", Robert).

La juxtaposition de procédés métaphoriques, loin d'additionner leurs avantages, donne plutôt l'impression de cumuler leurs défauts: si, dans l'extrait ci-dessus, on supprimait les termes propres à la discipline concernée (physique, Newton,...), le lecteur aurait sans doute bien des difficultés à comprendre de quoi ce texte traite. Bien sûr le paratexte est là pour clarifier le propos. Mais soulignons quand même

1 Idem, p. 45.

2 P. LÉVY, op. cit.

3 G. Chevalier, "Le zoo des particules", in Les Cahiers de Science \& vie, $\mathrm{n}^{\circ} 12$, Paris, décembre 1992. 
combien la juxtaposition de métaphores peut nuire à l'effet de chacune d'elles. La métaphore, gommant les niveaux logiques et amalgamant les éléments qu'elle met en relation, peut donner de la science, certes, une image riche et complexe mais une image qui néanmoins reste approximative. D'approximation en approximation, la juxtaposition excessive de métaphores aboutit finalement à une représentation floue et confuse, représentation limitant en fin de compte les possibilités inférentielles du destinataire.

Cette pratique -observée ici dans un discours de vulgarisationse rencontre fréquemment dans d'autres modes d'expression. Ainsi, l'écriture journalistique en fait abondamment usage et rares sont les journaux qui osent aujourd'hui s'en abstenir ${ }^{1}$. Cette tendance s'explique, en partie sans doute, par le pouvoir de camouflage de la métaphore qui, en donnant une vision approchée d'une réalité, permet de préserver l'ignorance de celui qui l'exhibe...

Mais ces dérives ne devraient pas conduire à jeter l'enfant avec l'eau du bain. La métaphore -je m'y suis longuement attardéconstitue une pièce essentielle de nos mécanismes cognitifs et communicationnels. Sans elle, comment pourrions-nous construire de nouvelles relations d'analogie, saisir le non représentable (par exemple l'espace à 3,4 ou à $n$ dimensions des mathématiciens) ou, tout bonnement, comprendre ce que j'essaie de vous dire?

Toutefois, la métaphore ne travaille pas en solitaire. Elle entretient -c'est du moins l'interprétation que j'ai donné aux écrits de Peirce- des liens étroits avec le diagramme et l'image. Dès lors, on peut soutenir que ceux-ci forment une condition d'emploi de celle-là. Sans dimension diagrammatique, la métaphore se confondrait avec l'image. S'il en était ainsi, le "code génétique" ou le "big bang" des physiciens nous apparaîtraient comme des images objectives (des données d'observation) et non comme des tentatives de comprendre notre environnement (micro ou macroscopique) par le transfert de notions appartenant à d'autres savoirs (la théorie de l'information pour le premier et l'expérience de la bombe explosive pour le second). De même, une métaphore construite sur un rapprochement sémantique inadéquat ou incongru ne parviendrait pas à faire ressortir l'image, le lien analogique visé (le "zoo" est-il un concept susceptible de métaphoriser la physique des particules?).

\footnotetext{
1 A ma connaissance, dans le paysage journalistique franco-belge, seul Le Monde semble résister à cette dérive.
} 
Enfin, et je terminerai par là, la communication d'un savoir ou d'une information doit s'interroger sur le genre d'icônes (image, diagramme et/ou métaphore) à exploiter. Ainsi, par exemple, un journaliste contraint d'expliquer au citoyen belge les enjeux d'un débat parlementaire portant sur la réforme des structures institutionnelles -et Dieu sait si ces dernières sont complexes- aura tout intérêt à recourir aux diagrammes qui permettent de montrer les (dés)équilibres et les rapports de force en jeu. Par contre, un parlementaire devant convaincre les membres de l'assemblée du bien fondé des idées de son parti, aura sans doute avantage à privilégier les procédés métaphoriques qui amalgament toujours quelque peu les éléments qu'ils rapprochent. Enfin, la réforme votée, on l'affublera, pour la postérité, du nom (et de la tête...) de son(ses) concepteur(s).

La métaphore n'est donc pas une panacée et elle mériterait, dans bien des cas, de laisser la place aux autres procédés iconiques. Mais, en la matière, on connaît le poids des habitudes (Tiens, encore une métaphore!). 